Revue internationale P.M.E.

Économie et gestion de la petite et moyenne entreprise

\title{
Une recherche-action pour diagnostiquer les pratiques de veille stratégique des PME
}

\section{François Brouard}

Volume 20, numéro 1, 2007

URI : https://id.erudit.org/iderudit/1008508ar

DOI : https://doi.org/10.7202/1008508ar

Aller au sommaire du numéro

\section{Éditeur(s)}

Presses de l’Université du Québec

ISSN

0776-5436 (imprimé)

1918-9699 (numérique)

Découvrir la revue

Citer cet article

Brouard, F. (2007). Une recherche-action pour diagnostiquer les pratiques de veille stratégique des PME. Revue internationale P.M.E., 20(1), 9-40.

https://doi.org/10.7202/1008508ar

\section{Résumé de l'article}

La recherche-action publiée dans un contexte de PME n'est pas fréquente, même si elle peut profiter aux PME en élaborant des savoirs actionnables et pertinents. L'article présente le processus d'une recherche-action, soit le développement d'un outil diagnostique des pratiques de veille stratégique auprès de PME. La présente recherche s'articule autour de la veille stratégique qui peut se définir comme un processus informationnel par lequel une organisation se met à l'écoute de son environnement pour décider et agir dans la poursuite de ses objectifs. La méthodologie de recherche retenue est la recherche-action et, plus particulièrement, le prototypage d'un système expert. L'objectif de la recherche est de sensibiliser les dirigeants d'une organisation aux activités de veille stratégique et ainsi contribuer à les faire progresser. Pour atteindre cet objectif, un outil diagnostique des pratiques de la veille stratégique auprès de petites et moyennes entreprises (PME) est développé. La structure du diagnostic s'attarde à cinq composantes principales, soit les types de veille, le contexte de la veille, l'organisation de la veille, le processus de la veille et la sécurité de l'information. Ces composantes se subdivisent pour former un grand total de 30 composantes. Dans le cadre du développement du prototype, 6 moyennes entreprises canadiennes et 33 experts sont mis à contribution lors des trois itérations menant à la dernière version du prototype. Les six PME ont fait l'objet d'autant d'études de cas.
Ce document est protégé par la loi sur le droit d'auteur. L'utilisation des services d'Érudit (y compris la reproduction) est assujettie à sa politique d'utilisation que vous pouvez consulter en ligne.

https://apropos.erudit.org/fr/usagers/politique-dutilisation/ 


\title{
Une recherche-action pour diagnostiquer les pratiques de veille stratégique des PME
}

François BROUARD'

Sprott School of Business

Carleton University

MOTS CLÉS

\section{Recherche-action - Veille stratégique - Outils de gestion \\ Performance - Système expert}

\begin{abstract}
L'AUTEUR
Françols Brouard est détenteur d'un doctorat en administration (DBA) de l'Université du Québec à Trois-Rivières et professeur en fiscalité et en comptabilité financière à la Sprott School of Business, Carleton University, à Ottawa. Comptable agréé de formation, François Brouard s'intéresse notamment dans le cadre de ses recherches aux PME, à l'entrepreneuriat, à la veille stratégique et à la gouvernance. Coordonnées: Sprott School of Business, Carleton University, 1125 Colonel By Drive, Ottawa, Ontario, K1S 5B6. Courriel : <francois_brouard@ carleton.ca>.
\end{abstract}

\section{RÉSUMÉ}

La recherche-action publiée dans un contexte de PME n'est pas fréquente, même si elle peut profiter aux PME en élaborant des savoirs actionnables et pertinents. L'article présente le processus d'une recherche-action, soit le développement d'un outil diagnostique des pratiques de veille stratégique auprès de PME. La présente recherche s'articule autour de la veille stratégique qui peut se définir comme un processus informationnel par lequel une organisation se met à l'écoute de son environnement pour décider et agir dans la poursuite de ses objectifs. La méthodologie de recherche retenue est la recherche-action et, plus particulièrement, le prototypage d'un système expert. L'objectif de la recherche est de sensibiliser les dirigeants d'une organisation aux activités de veille stratégique et ainsi contribuer à les faire progresser. Pour atteindre cet objectif, un outil diagnostique des pratiques de la veille stratégique auprès de petites et moyennes entreprises (PME) est développé. La structure du diagnostic s'attarde à cinq composantes principales, soit les

1. L'auteur aimerait remercier Louis Raymond pour les nombreux conseils lors de la réalisation de cette recherche et les évaluateurs pour leurs remarques pour améliorer son travail. 
types de veille, le contexte de la veille, l'organisation de la veille, le processus de la veille et la sécurité de l'information. Ces composantes se subdivisent pour former un grand total de 30 composantes. Dans le cadre du développement du prototype, 6 moyennes entreprises canadiennes et 33 experts sont mis à contribution lors des trois itérations menant à la dernière version du prototype. Les six PME ont fait l'objet d'autant d'études de cas.

\section{ABSTRACT}

Action research published in the SME context is not frequent, even if it could be beneficial for SME to develop actionable and relevant knowledge. The paper presents an action research process for the development of a diagnostic tool on environmental scanning practices of SME. The research is articulated around the environmental scanning theme defined as an informational process by which an organization stays attuned to its environment in order to make decisions and then act in pursuit ot its objectives. The methodology used is an action research and more specifically prototyping of an expert system. The purpose of the research is to help managers improve their awareness of environmental scanning practices and contribute to increased use of these practices. To reach that objective, a diagnostic tool of environmental scanning practices of SME is developed. The diagnosis structure looks at five main components: scanning types, scanning context, scanning organization, scanning process, and information security. Those components are divided to obtain a grand total of 30 components. During the prototype development, 6 medium Canadian firms and 33 experts are involved during three iterative processes to obtain the last version of the prototype. Six SME were subject of case studies.

\section{RESUMEN}

Las publicaciones sobre investigaciones prácticas en el contexto de PyMEs no son frecuentes, aun cuando pudiera ser beneficioso para las PyMEs desarrollar conocimientos relevantes y prácticos. Este trabajo presenta un proceso de investigación práctica para el desarrollo de una herramienta de diagnóstico para las esas desarrollas empresas desarrollas uando sería prácticas de escaneo ambiental de las PyMEs. La investigación se articula alrededor del tema de escaneo ambiental definido como un proceso informacional mediante el cual una organización se mantiene a tono con su medio para poder tomar decisiones y luego actuar para lograr sus objetivos. La metodología utilizada es una investigación práctica y, en específico, la creación de un prototipo de un sistema experto. El propósito de la investigación es ayudar a los gerentes a aumentar su conocimiento sobre las prácticas de escaneo ambiental y contribuir a un mayor uso de estas prácticas. Para alcanzar ese objetivo, se desarrolla una herramienta de diagnóstico de prácticas de escaneo ambiental de PyMEs. La estructura de diagnóstico analiza cinco componentes principales : tipos de escaneo, contexto del escaneo, organización del escaneo, proceso de escaneo y seguridad de la información. Esos componentes se dividen para obtener un total general de 30 componentes. Durante el desarrollo del prototipo, 6 medianas empresas canadienses y 33 expertos participan durante tres procesos iterativos para obtener la versión final del prototipo. Seis PyMEs fueron objeto de estudios de caso.

Revue internationale P.M.E., vol. 20, nº 1, 2007

(C) 2007 - Presses de l'Université du Québec

Édifice Le Delta I, 2875, boul. Laurier, bureau 450, Québec, Québec G1V 2M2 • Tél.: (418) 657-4399 - www.puq.ca

Tiré de: Revue internationale P.M.E., vol. 20, $\mathrm{n}^{\circ} 1$, sous la direction de Louis Raymond - PME2001N Tous droits de reproduction, de traduction et d'adaptation réservés 


\section{ZUSAMMENFASSUNG}

Publizierte Resultate aus der Aktionsforschung aus Kontext der KMU sind eher selten, obwohl sie für kleine und mittlere Unternehmen brauchbares Handlungswissen und aussagekräftige Erkenntnisse hervorbringen. Der Artikel umschreibt den Forschungsprozess zur Erarbeitung eines diagnostischen Analysemodells der Business-Intelligence Praktiken in KMUs. Unter Business-Intelligence wird im vorliegenden Artikel ein Informationsprozess verstanden, mit dem sich das Unternehmen eingehend mit der eigenen Umwelt beschäftigt, um Entscheide zu treffen und entsprechend den Zielvorgaben zu handeln.

Das methodische Vorgehen orientiert sich weitgehend an der Aktionsforschung, welche als Prototyp eines Expertensystems gestaltet wurde. Die Zielsetzung der Forschung liegt darin, den Führungskräften eines Unternehmens die Aktivitäten im Rahmen der Business-Intelligence näher zu bringen und ihnen anschliessend dabei zu helfen, sich zu verbessern. Um dies zu erreichen, wurde ein diagnostisches Analysemodell der Business-Intelligence Praktiken von kleinen und mittleren Betrieben entwickelt. Das Diagnosemodell basiert auf der Ausprägung, den Zusammenhängen, die Organisation, dem Prozess und der Informationssicherheit von Business-Intelligence. Diese fünf Komponenten werden weiter unterteilt, womit ein Raster von 30 Bausteinen entsteht. Zur Erstellung des Prototyps haben sechs Fallstudien mittelgrosser kanadischer Unternehmen und 33 Experten in drei sich wiederholenden Durchläufen beigetragen.

\section{Introduction}

Pour relever les défis posés par la complexité grandissante, les changements rapides et les incertitudes de plus en plus nombreuses, les petites et moyennes entreprises (PME) doivent devenir des entreprises agiles de classe mondiale pour survivre et s'adapter aux changements en émergence (Julien, 1994; Kasarda et Rondill, 1998; Raymond, 2000). Comme l'adaptation aux changements de l'environnement est une nécessité de tous les instants pour faire face à ces défis, l'identification des changements le plus efficacement possible est un problème de gestion méritant une étude plus approfondie.

La veille stratégique est reconnue comme étant un dispositif au moyen duquel on peut connaître l'environnement d'affaires et anticiper les changements (Aguilar, 1967; Lesca, 2003). La veille stratégique peut se définir comme un processus informationnel par lequel une organisation se met à l'écoute de son environnement pour décider et agir dans la poursuite de ses objectifs. Ce processus s'insère dans l'ensemble des systèmes d'information et vise à permettre aux dirigeants de mieux piloter dans la tempête de changements.

La problématique de départ concerne la manière dont un intervenant interne ou externe peut aider les organisations en matière de veille stratégique. Il ressort d'études antérieures que les entreprises, en particulier les

Revue internationale P.M.E., vol. 20, nº 1, 2007 
PME, doivent tout d'abord être sensibilisées à la veille stratégique et aux avantages que procurent ces activités (Bulinge, 2002, 2003; CNRC-ICIST, 1999; Larivet, 2002; Lesca et Raymond, 1993). Cette sensibilisation est une condition nécessaire aux décisions d'investissement et d'implantation d'activités de veille stratégique. En effet, sans cette prise de conscience, les PME risquent de négliger ce type d'activités. Les organisations doivent aussi posséder des moyens pour les aider à progresser en leur permettant d'évaluer l'état actuel des pratiques qu'elles utilisent.

L'axe de recherche privilégié est donc l'établissement d'un diagnostic des activités de veille stratégique pour comprendre les pratiques existantes et rendre explicites les processus. Ce diagnostic devrait se faire à l'aide d'outils à développer. Ainsi, l'évaluation de la performance par une mesure de la qualité du processus de veille stratégique et le diagnostic des pratiques de veille sont les éléments privilégiés pour répondre aux besoins des organisations et intervenir en leur fournissant des recommandations (Lesca, 1994; Lesca et Rouibah, 1997).

Pour y parvenir, la recherche-action représente une méthodologie méritant d'être étudiée davantage dans le contexte de la PME et des systèmes d'information afin de produire des savoirs actionnables et pertinents (Avenier, 2004; Baskerville et Myers, 2004). En l'absence d'un cadre conceptuel généralement reconnu au niveau de la veille stratégique, il convient de recourir à une stratégie de recherche plus qualitative et basée sur le terrain (Audet, 1998; Bergeron, 1997, 2000; Ganesh, Miree et Prescott, 2003). En effet, l'approche qualitative est une «méthodologie appropriée pour étudier un concept émergent et mal défini comme l'est le concept de veille stratégique» (Bergeron, 2000, p. 5).

Même si elle est jugée pertinente, il est à noter que la recherche-action ne semble pas encore très populaire dans les recherches publiées en Amérique $\mathrm{du}$ Nord relativement aux systèmes d'information, une étude la situant à moins de 1 \% des recherches (Baskerville et Wood-Harper, 1996; Lau, 1999). Une recension n'indique également aucun article dans la Revue internationale $P M E$ relatant une expérience de recherche-action; les articles les plus récents ayant été les articles d'Avenier (2004) et de Schmitt (2004).

L'objectif de la recherche est de sensibiliser les dirigeants d'une organisation aux activités de veille stratégique et ainsi contribuer à les faire progresser. La sensibilisation se réfère à une meilleure connaissance d'un concept, comme celui de la veille stratégique. La progression signifie un mouvement en avant, soit la poursuite ou l'amélioration des pratiques de veille stratégique d'une entreprise. Les PME présentant des caractéristiques 
spécifiques, il importe de créer des outils propres au contexte des PME (GREPME, 1997). Pour atteindre cet objectif, un outil diagnostique orienté vers les PME, sous forme de système expert, est développé.

Cet article vise à présenter le développement d'un outil diagnostique des pratiques de veille stratégique auprès de PME. Il comprend cinq sections: 1) la recherche-action et le prototypage;2) le cadre conceptuel de la veille stratégique;3) le choix et l'architecture du système expert;4) une discussion des résultats obtenus; 5) les retombées de cette recherche. Une conclusion complète le tout.

\section{Recherche-action et prototypage}

Puisque les questions visées par la présente recherche portent sur la compréhension et l'intervention, la méthodologie de recherche retenue est la recherche-action et, plus particulièrement, le prototypage d'un système expert. Greenwood et Levin (1998) ancrent la recherche-action dans le paradigme du pragmatisme et la théorie des systèmes. La recherche-action se fonde sur la «conviction que la recherche et l'action peuvent être réunies » (Lavoie, Marquis et Laurin, 1996, p. 61). L'actionnabilité de la recherche au niveau de la PME est appuyée par Avenier (2004).

\subsection{Définition, finalités et caractéristiques de la recherche-action}

Il semble exister une certaine confusion dans la définition de la rechercheaction (Baskerville, 1999; Dolbec, 1998). Il est néanmoins essentiel d'en fournir une qui soit reconnue (Baskerville et Wood-Harper, 1996). «La recherche-action aide simultanément la résolution de problèmes pratiques et l'amélioration des connaissances, en améliorant les compétences des acteurs dans un esprit de collaboration et d'échanges face à une situation donnée, en utilisant un processus cyclique visant une meilleure compréhension de la situation et des changements d'un système social et se déroulant dans un cadre mutuellement acceptable par les acteurs au point de vue éthique» (Hult et Lennung, 1980, p. 247; traduction libre).

Pour Dolbec (1998), la finalité de la recherche-action est triple et comprend les pôles recherche, action et apprentissage. Le pôle recherche «représente l'utilisation d'une approche méthodologique rigoureuse pour guider, éclairer le processus de résolution de problèmes tout au long de son déroulement et en évaluer l'impact» (Dolbec, 1998, p. 486). Le pôle action « représente l'intervention choisie pour initier un changement au sein d'une situation concrète» (Ibid.,p. 486). Le pôle apprentissage vise les apprentissa- 
ges effectués par ceux qui veulent «comprendre la situation et son contexte, le contenu de l'intervention et l'apport du processus de recherche-action lui-même vu comme une stratégie de changement» (Ibid., p. 486).

À la suite d'une revue de la documentation, Lavoie, Marquis et Laurin (1996) résument les caractéristiques de la recherche-action en les regroupant selon qu'il s'agit de conditions critiques ou non critiques de la rechercheaction. Les conditions critiques d'une recherche-action sont d'avoir pour origine des besoins sociaux réels, d'être menée en milieu naturel de vie, de faire participer le chercheur et les partenaires, d'être flexible, d'établir une communication systématique et de s'autoévaluer. Les conditions non critiques sont que la recherche-action est à caractère empirique, en lien dynamique avec le vécu, caractérisée par une démarche novatrice et une forme de gestion collective, où le chercheur est aussi acteur et où l'acteur est aussi chercheur. Ces caractéristiques correspondent à celles relevées dans les recherches en gestion et celles portant sur les systèmes d'information (Baskerville, 1999; Baskerville et Wood-Harper, 1998; Eden et Huxman, 1996; Mansell, 1991).

Comme toute autre méthode, la recherche-action est plus valide à l'intérieur d'un domaine idéal de question de recherche (Baskerville et WoodHarper, 1996). Le domaine idéal de la recherche-action s'inscrit dans un contexte où 1) le chercheur est un participant actif qui s'attend à des bénéfices pour lui-même et l'organisation impliquée, 2) la connaissance obtenue peut être immédiatement appliquée et 3) la recherche est un processus cyclique liant la théorie et la pratique (Baskerville, 1999; Baskerville et Wood-Harper, 1996). Ainsi, la fusion entre la recherche et la pratique («praxis») permet d'obtenir des résultats pertinents (Baskerville et Wood-Harper, 1996).

L'objectif visé par la présente recherche est à la fois de comprendre les pratiques relatives à la veille stratégique et d'intervenir auprès des entreprises pour amener une progression à cet égard. Les buts de la recherche-action exprimés par Lavoie, Marquis et Laurin (1996) sont de changer des situations existantes, de comprendre les pratiques, d'évaluer, de résoudre des problèmes, de produire des connaissances et d'améliorer des situations existantes. Une comparaison des objectifs de la présente recherche et des objectifs de la recherche-action permet de conclure que cette approche est justifiée pour cette étude appliquée.

Tout comme il existe une diversité de définitions, il existe une diversité de modèles de la recherche-action (Lavoie, Marquis et Laurin, 1996). Le principal modèle de recherche-action retenu est celui de Susman et Evered (1978); il s'agit de l'un des modèles les plus fréquemment utilisés pour décrire la recherche-action (Baskerville, 1999). Selon ce modèle, la rechercheaction comprend cinq étapes, soit le diagnostic, la planification de l'action, 
la réalisation de l'action, l'évaluation et la définition de connaissances nouvelles s'insérant dans une infrastructure d'un système-client (voir figure 1; Baskerville, 1999; Lavoie, Marquis et Laurin, 1996; Susman et Evered,1978). Il s'agit d'une démarche cyclique et perpétuelle, qui peut se visualiser sous la forme d'une spirale.

\section{Figure 1}

\section{Modèle d'étapes de recherche-action}

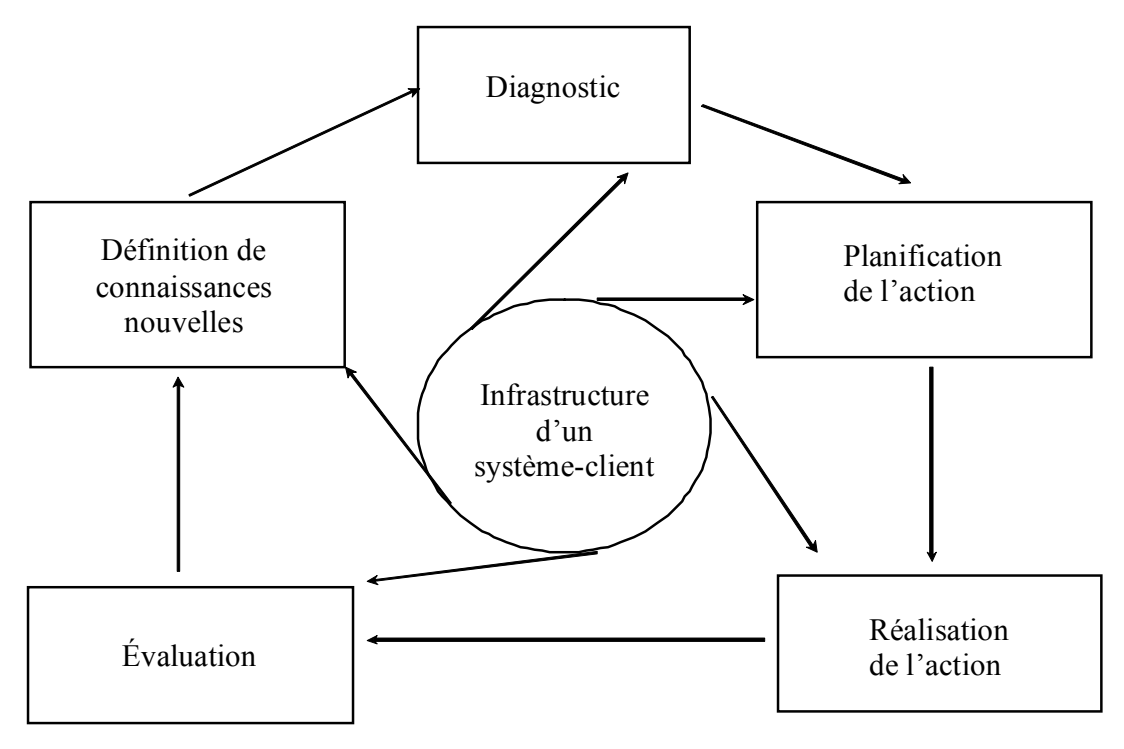

Source: Susman et Evered, 1978.

\subsection{Variante retenue de la recherche-action: le prototypage}

Il existe plusieurs variantes de la recherche-action (Lavoie, Marquis et Laurin, 1996). Baskerville (1999) en mentionne 10: le prototypage, la méthodologie des systèmes souples, la méthode canonique (canonical), la méthode ETHICS, la multiview, l'action science, l'observation participante, l'action learning, le travail clinique (clinical field work) et la consultation. La variante de la recherche-action retenue est le prototypage. Certains auteurs qualifieraient plutôt notre démarche de recherche ingénierique, qui peut être vue comme un sous-ensemble de la recherche-action lors de la construction d'artefacts, soit notre prototype (Chanal, Lesca et Martinet, 1997). 
Le prototypage se définit comme une «approche pour construire des systèmes d'information qui utilisent des prototypes» (Beynon-Davies, Tudehope et Mackay, 1999, p. 108; traduction libre). Un prototype est un modèle de travail d'un système d'information (ou d'une partie de celui-ci) dans une version préliminaire. Le prototypage vise à «connaître le plus de chose sur le comportement de ce système par expérimentation dynamique» (Rubiello, 1997, p. 122). En somme, il s'agit de «faire passer le système d'un haut état d'abstraction (dans la tête de l'utilisateur) à un état de prototype réel (non abstrait) et donc réfutable et améliorable» (Ibid., p. 135). «Le cycle d'inspection-discussion-modification est répété plusieurs fois jusqu'à ce que l'utilisateur soit satisfait du système » (Beynon-Davies, Tudehope et Mackay, 1999, p. 108; traduction libre).

À partir de la classification des caractéristiques permettant de positionner les formes de recherche-action de Baskerville (1999), il est possible de positionner le prototypage. Le prototypage est ainsi un modèle de processus itératif avec une structure rigoureuse où l'implication du chercheur est celle d'un collaborateur ou d'un facilitateur et dont le but principal est le design de système (Baskerville et Wood-Harper,1998). Dans notre cas, l'implication du chercheur se situe à deux niveaux, soit celui d'un facilitateur pour amener le changement auprès des PME, mais aussi celui d'un client à titre de chercheur désirant développer un outil.

Le prototypage est une méthode utilisée dans la recherche-action appliquée aux systèmes d'information. Les éléments favorisant le développement réussi d'un système par le prototypage justifient concrètement le choix effectué. Baskerville (1999) énumère ces éléments, soit la présence d'un artefact immédiat permettant de rendre visibles et tangibles les résultats, le développement d'une expertise, la validation constante et permanente des spécifications à travers la réalité des réviseurs, l'amélioration des communications entre les utilisateurs et le designer, la compréhension partagée, la participation accrue des utilisateurs dans le processus de design et l'acceptation du nouveau système. Le recours à un prototype permet aussi de sensibiliser les participants en leur démontrant de manière opérationnelle et pratique un concept abstrait, comme c'est le cas avec le concept de veille stratégique (Raymond et Lesca, 1995).

\section{Cadre conceptuel de la veille stratégique}

Peu importe sous quel angle la veille stratégique est abordée, l'enjeu central demeure la survie de l'entreprise, qui est menacée par l'incertitude créée dans l'environnement. La prise de décision pour assurer cette survie est au cœur des activités de veille. Quatre types de veille peuvent être soulignés, 
soit la veille technologique, la veille concurrentielle, la veille commerciale et la veille sociétale. La veille stratégique englobe l'ensemble des veilles particulières. Notons que chaque type de veille permet de combler des besoins particuliers.

Comme il s'agit d'un sujet relativement nouveau en gestion (une trentaine d'années), il n'y a pas de cadre conceptuel généralement reconnu de la veille stratégique, ce qui constitue une lacune (Bergeron, 1997; Choudhury et Sampler, 1997; Ganesh, Miree et Prescott, 2003; Zou et Cavusgil, 1996). Ganesh, Miree et Prescott (2003) soulignent cependant l'importance de posséder un cadre conceptuel et de faire avancer ce champ disciplinaire émergent. Il faut signaler que plusieurs recherches récentes proposent des meilleures pratiques et des cadres conceptuels sur la veille et s'intéressent à la formalisation de la recherche à l'égard de ce thème (Choo, 1999; Ganesh, Miree et Prescott, 2003; Jacob, Julien et Raymond, 1997; Liu, 1998).

S'appuyant sur une synthèse des travaux antérieurs, depuis Aguilar (1967), il est possible d'articuler une vision de la veille stratégique. Cette articulation permet d'organiser et de structurer l'outil diagnostic selon un cadre conceptuel. Le développement de la base de connaissances du système expert a permis d'effectuer une synthèse des connaissances portant sur les pratiques de veille stratégique.

Le processus de la veille stratégique peut se décrire comme un système qui se compose lui-même de sous-systèmes. Ces systèmes et sous-systèmes de la veille stratégique sont influencés par des flux d'information provenant du macroenvironnement, des acteurs et par l'organisation elle-même. Le macroenvironnement comporte des dimensions démographique, économique, technologique, politique, juridique, écologique, géophysique et socioculturelle. Les acteurs ou détenteurs d'enjeux identifiés sont les clients, les fournisseurs, les employés, les syndicats, les partenaires, les compétiteurs, les gouvernements, les médias, les groupes de pressions et les réseaux. L'environnement interne de l'organisation correspond à ses ressources, à sa culture, à ses stratégies, à sa direction et à sa structure. Les dimensions de l'environnement interne ont une influence sur des sous-systèmes liés aux ressources, à la culture, à la direction et à la structure de la veille. La figure 2 a illustre les systèmes du processus de la veille stratégique et les influences qui les affectent.

Le système principal du processus de la veille stratégique se décompose en trois éléments, soit les intrants, les cycles et les extrants. Les intrants renvoient aux nombreux besoins manifestés par les utilisateurs. Le premier cycle concerne la collecte de l'information avec les phases de planification, de recueil, d'analyse et de diffusion. Le second cycle vise la protection de cette information et comprend la planification, l'analyse des vulnérabilités, 
l'évaluation des risques et des menaces ainsi que les mesures de protection. Chacun de ces deux cycles est suivi par un volet apprentissage. Nous avons donc la dimension offensive de la collecte et la dimension défensive de la protection. Dans la figure 2b, qui illustre ces deux cycles, les extrants correspondent aux produits orientés vers les décisions et l'action. Selon les phases du cycle de la veille stratégique, ces produits peuvent être des données, de l'information ou des connaissances.

FIGURE 2A

\section{Processus de la veille stratégique et flux}

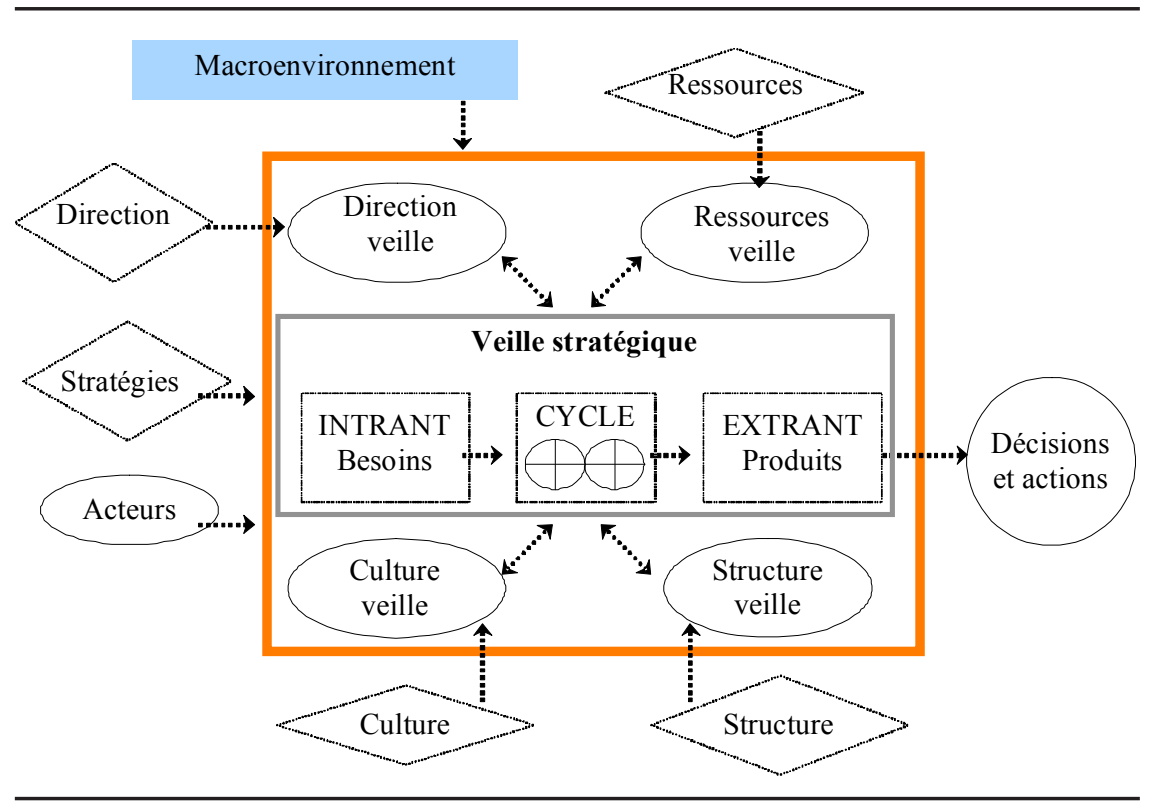

Revue internationale P.M.E., vol. 20, nº 1, 2007 
FIGURE 2B

\section{Cycle de la veille stratégique}

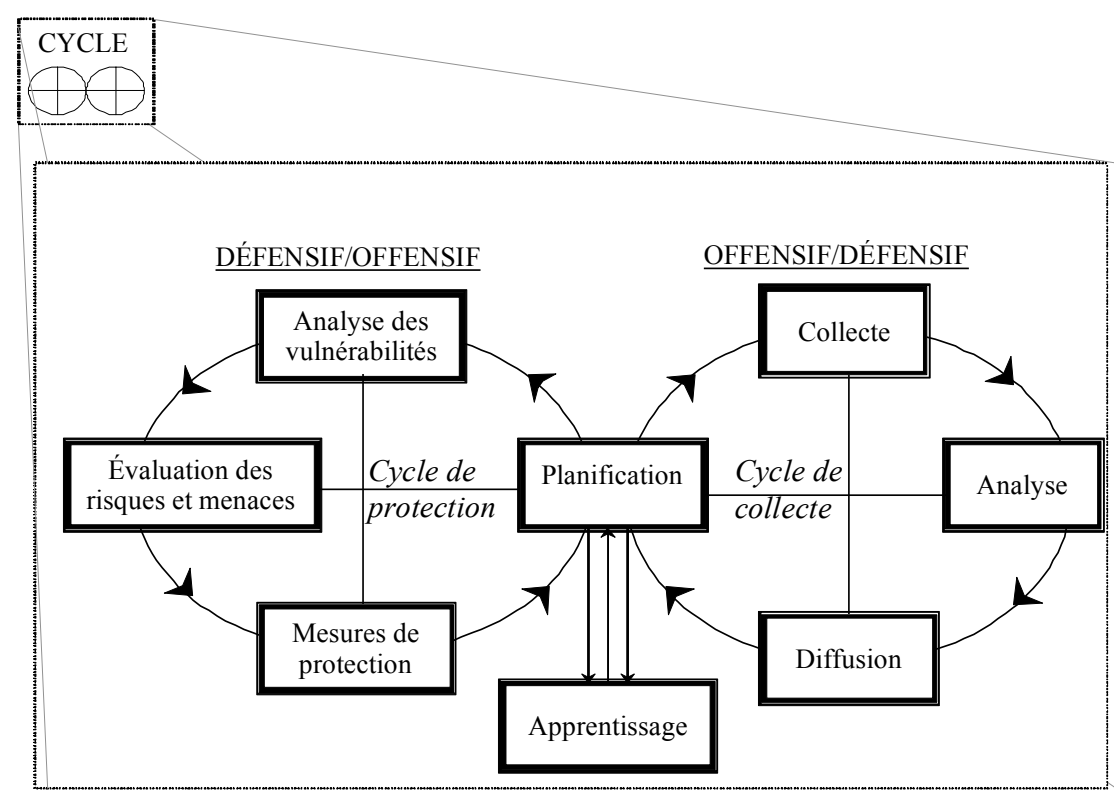

Ce cadre conceptuel s'appuie sur diverses théories dont la théorie de l'organisation industrielle, la théorie basée sur les ressources, la théorie économique et la théorie de la contingence (Choudhury et Sampler, 1997; Liu, 1998; Zou et Cavusgil, 1996). Ce cadre représente une synthèse des travaux antérieurs, incluant les meilleures pratiques décrites dans la littérature. Cette conception de la veille stratégique intègre les principales composantes des cadres conceptuels proposés par Auster et Choo (1994), Choo (1999, 2001), Elenkov (1997), Jacob, Julien et Raymond (1997), Julien et al. (1999), Liu (1998) et Vandenbosch et Huff (1997).

\section{Choix et architecture du système expert}

Un système expert est un programme informatique permettant de créer des solutions à des problèmes en utilisant la connaissance humaine emmagasinée dans une base de connaissances. Il se compose de quatre éléments fondamentaux, soit une base de connaissances, un moteur d'inférence, une interface de l'utilisateur et une interface d'acquisition de connaissances (Benfer, Brent et Furbee, 1991). Le développement est d'abord axé sur l'élaboration de la base 
de connaissances et des règles qui régissent le fonctionnement du système expert et ensuite sur la conception d'un questionnaire permettant d'alimenter ce système expert en variables afin d'assurer le fonctionnement des règles.

Ainsi, l'outil diagnostique développé prend la forme d'un outil informatisé et, plus particulièrement, d'un système expert grâce auquel il est possible de poser un diagnostic des pratiques de veille stratégique de PME. L'outil applique la connaissance humaine à un domaine spécifique afin de résoudre des problèmes. Par définition, un prototype est un modèle de travail dans une version préliminaire et son développement n'a donc pas atteint sa pleine maturité.

\subsection{Pertinence du choix d'un système expert}

Le système expert correspond à une des applications de l'intelligence artificielle et représente l'une des applications les plus courantes de l'intelligence artificielle (Awad, 1996). L'intelligence artificielle est une «science qui rend les machines capables de faire des choses qui requièrent de l'intelligence si elles étaient effectuées par des humains» (Awad,1996, p. 5 ; traduction libre). Ainsi, l'intelligence artificielle peut être vue comme une suite de procédures qui permet à un ordinateur de voir, entendre, comprendre, raisonner ou atteindre des résultats similaires à ceux qu'une personne atteindrait en transformant ses connaissances (Awad, 1996).

La complexité du contexte, le grand nombre de variables à considérer et la disponibilité de moyens technologiques peu coûteux amènent l'utilisation de technologie de l'information et de communication. Au regard de la problématique de recherche énoncée et du contexte de celle-ci, le système expert possède plusieurs avantages comparativement aux autres types de systèmes d'information pour appliquer la solution envisagée. La problématique crée un besoin au niveau d'un diagnostic orienté vers les dirigeants. La sensibilisation des utilisateurs, la formalisation de la connaissance et le transfert de connaissances font également partie des objectifs visés par cet outil. En outre, il ne faut pas perdre de vue que le contexte de la problématique correspond à un système complexe avec des décisions non structurées. Comme les entreprises ne possèdent pas ou peu de données sur leurs activités de veille stratégique, il apparaît nécessaire de faire appel à un outil qui effectue la collecte de ces données.

S'agissant de l'établissement d'un diagnostic, Turban et Aronson (1998) citent deux types de systèmes répondant à ce besoin, soit les systèmes experts et les réseaux neuronaux. Les réseaux neuronaux, qui font partie des applications de la science informatique d'intelligence artificielle, sont également des 
systèmes produisant un diagnostic (Turban et Aronson, 1998). Toutefois, ces systèmes sont orientés vers la reconnaissance de «patterns » et s'alimentent à partir de cas historiques. Awad (1996) précise que le fonctionnement des réseaux neuronaux oblige l'utilisateur à fournir les données de départ et à définir les résultats souhaités afin que le système génère le processus à suivre. Par comparaison, une personne utilisant un système expert fournit les données de départ et les règles du processus à suivre et les systèmes experts fournissent des explications du processus suivi, en plus d'un diagnostic. La complexité de développement et le manque de capacité au plan explicatif constituent donc des limites importantes dans le développement de systèmes neuronaux.

Enfin, les systèmes experts semblent répondre à l'ensemble des préoccupations de la problématique et de son contexte. En plus de donner des conseils et des explications sur leur provenance, ce qui est important compte tenu de l'état des connaissances et du degré de sensibilisation, ils jouent le rôle de conseillers experts auprès des utilisateurs (O'Brien, 2001). Le choix d'un système expert est également judicieux dans un contexte de PME (Hebert et Bradley, 1993; Martin et al.,1991). Au Québec, une étude a révélé que les systèmes experts sont passés du stade expérimental au stade industriel (Novasys, 1991, cité dans Lyoussoufyine et Raymond, 1998). Le développement du système doit toutefois tenir compte de la spécificité des PME et de l'importance du dirigeant (Moreau et al., 2002; Moreau, Raymond et Turcot, 2003).

L'utilisation d'un système expert offre plusieurs avantages. Au nombre de ceux-ci, notons l'amélioration de la productivité, la formation du personnel, la conservation d'une expertise rare, l'amélioration de la performance du personnel, la disponibilité d'une expertise relativement abordable, la standardisation de l'approche à un problème, l'amélioration de la qualité de la décision, l'utilisation d'information incomplète ou incertaine, la réduction du temps de réponse, l'intégration de l'opinion de plusieurs experts et le transfert de connaissances dans des régions éloignées (Awad, 1996; Turban et Aronson, 1998).

Lesca et Rouibah (1997) et Lesca (2000) relèvent quelques outils informatisés développés pour la veille stratégique, même s'il ne s'agit pas d'outils visant la réalisation d'un diagnostic. Ces outils portent sur la pertinence (PERTINENCE), le ciblage (CIBLE), la sélection (SELECT, OASIS) et la création de sens (PUZZLE). Des consultants ont également développé divers outils ou méthodologies pour des fins spécifiques.

Parmi les outils informatisés développés, le logiciel FENNEC est un instrument conçu sous forme de système expert pour sensibiliser les dirigeants 
d'une PME et les aider à établir un diagnostic rapide de veille stratégique en vue d'un progrès éventuel (Lesca, 1991; Lesca et Raymond, 1993; Lesca et Rouibah, 1997; Raymond et Lesca, 1995). Toutefois, FENNEC «ne montre pas comment passer de l'état actuel des lieux à un état préférable ( Lesca et Rouibah, 1997, p. 123). Après avoir utilisé le système expert FENNEC, Lesca et Raymond (1993, p. 63) suggèrent la conception et la réalisation d'un «système expert qui fournit non seulement un diagnostic et une orientation en matière de veille stratégique, mais aussi des méthodes et des outils appropriés pour passer de l'état actuel des lieux à un état jugé préférable (par exemple, pour relever les besoins informationnels propres à l'entreprise pour déterminer les moyens de satisfaire ces besoins)».

La société française Egideria propose également un outil pour effectuer un audit de l'intelligence économique; il s'agit d'un prototype disponible gratuitement sur leur site Web et dont la dernière mise à jour remonte à 2001. Quatre modules sont prévus: l'audit des besoins stratégiques et des moyens de l'intelligence économique, un audit du processus de l'intelligence économique, un audit des organisations d'intelligence et un audit des outils; seulement les deux premiers modules sont opérationnels.

Bien qu'ils soient le fruit d'efforts louables vers une meilleure veille, les outils du laboratoire de recherche du groupe Lesca (1991; FENNEC) et d'Egideria (2003) présentent des lacunes quant à leur performance. Ainsi, la conception de ces deux outils est relativement limitée sur le plan des questions posées pour établir le diagnostic, ce qui empêche de nuancer les recommandations. Par exemple, l'ensemble des questions tient en 4 ou 5 pages au lieu de 32 avec le système expert développé. Les diagnostics semblent faire abstraction de la situation particulière d'une organisation spécifique et il y a peu de correspondance entre le niveau des pratiques et les besoins et ressources d'une organisation. En outre, la terminologie européenne et le style spécialisé ne semblent pas nécessairement convenir à la compréhension des pratiques nord-américaines par des dirigeants de PME. Par conséquent, il peut être difficile pour un dirigeant non initié à la veille d'intervenir concrètement pour améliorer sa situation. De plus, l'analyse des pratiques de veille ne semble pas tenir compte de la globalité des pratiques de l'entreprise. Les recommandations sont présentées en quelques paragraphes seulement et aucune explication n'est fournie au lecteur pour l'aider à en comprendre l'origine. En somme, l'aide permettant au lecteur de mieux comprendre les différentes pratiques et de sensibiliser est limitée. 


\subsection{Architecture de l'outil diagnostique et méthodologie}

L'architecture du système expert repose sur un questionnaire grâce auquel des données sont recueillies sur chaque entreprise et saisies ensuite dans une banque de données. Le système expert développé utilise la coquille Visual Rule Studio 2.5 de Rules Machine Corporation et le langage de programmation associé à celui-ci, Visual Basic 6.0 de Microsoft. Le système expert comprend l'interface de l'utilisateur, le moteur d'inférence et la base de connaissances; ce système expert rend un diagnostic des pratiques de veille stratégique pour l'entreprise sous étude.

Des recommandations visant à améliorer les pratiques de veille stratégique viennent compléter les feux de circulation et soutiennent l'entreprise dans son action. Des explications générales sur la veille permettent au lecteur du rapport de diagnostic de mieux comprendre les concepts de la veille stratégique. Signalons que la programmation a permis de développer un ensemble de règles; au total, le système expert sur la veille stratégique en contient 588 . Une règle peut se définir comme un énoncé de la connaissance et prendre la forme d'un appariement entre une condition et une action. Par exemple, la formulation pourrait se lire ainsi: «IF la condition se produit THEN l'action X apparaît ELSE sinon l'action Y apparaît» («IF-THEN-ELSE»; Turban et Aronson, 1998). La figure 3 illustre l'architecture du système expert sur la veille.

Basée sur le cadre conceptuel, la structure du diagnostic comprend cinq principales composantes, soit les types de veille, le contexte de la veille, l'organisation de la veille, le processus de la veille et la sécurité de l'information. Ces composantes se subdivisent pour former un grand total de 30 composantes (voir figure 4). Le diagnostic se présente sous la forme d'une série de feux de circulation (traffic lights), indiquant trois niveaux de performance (rouge, jaune, vert). Cette codification à trois niveaux est reconnue et sa compréhension est aisée. Des recommandations visant à améliorer les pratiques de veille stratégique viendront compléter l'image de la situation ainsi que le diagnostic. 
Figure 3

Architecture du système expert sur la veille

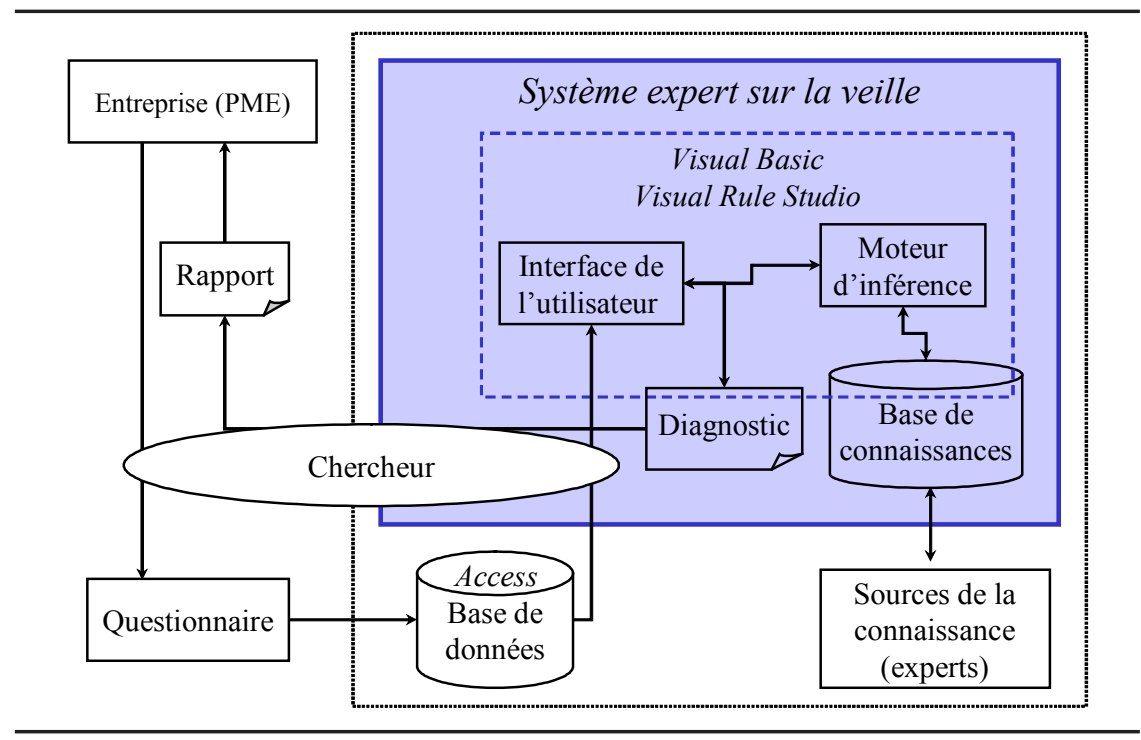

FIGURE 4

Structure du diagnostic de la veille stratégique

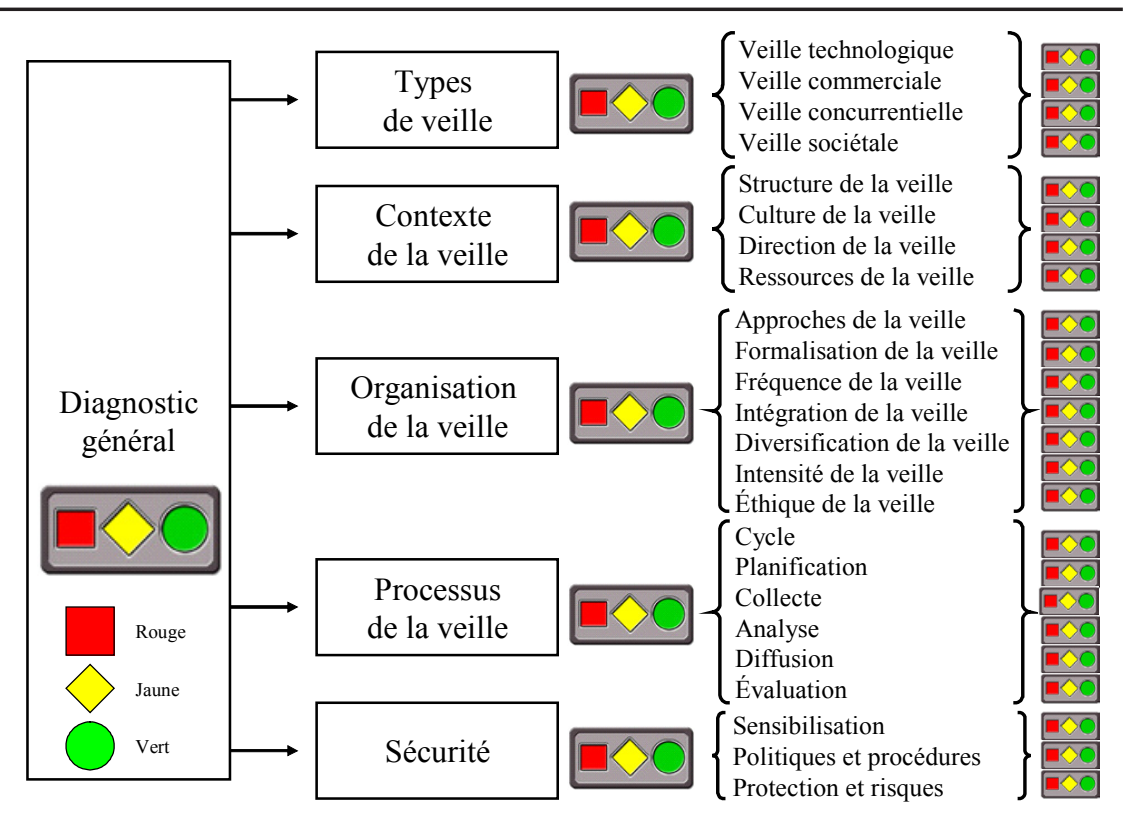

Revue internationale P.M.E., vol. 20, nº 1, 2007 
L'étude de cas est la tactique privilégiée, notamment pour tester les différentes versions du prototype, pour enrichir la base de connaissances et pour procéder à l'évaluation du système expert développé. Le choix retenu est l'étude de plusieurs entreprises (étude de cas multiples), afin de valider l'outil diagnostique avec une diversité de situation (Eisenhardt, 1989; Yin, 1994). L'unité d'analyse correspond à l'entreprise et en particulier à l'ensemble des activités liées à la veille stratégique. Trois principaux outils de collecte ont été choisis parmi les méthodes de recherche; il s'agit de l'entrevue, du questionnaire et de l'analyse documentaire.

L'entrée des données pour établir le diagnostic des pratiques de veille stratégique d'une entreprise se fait à partir d'un questionnaire, qui permet de répertorier les indicateurs des pratiques de veille stratégique des entreprises. Les réponses constituent en fait la matière première du diagnostic en alimentant le système expert.

La méthodologie de recherche préconisée requiert deux populations différentes, soit des entreprises et des experts. Les entreprises servent à la rédaction des études de cas tests pour l'évaluation de leurs pratiques de veille stratégique tandis que les experts contribuent à valider l'outil diagnostique développé lors des différentes itérations durant le développement. Dans le cadre du développement du prototype, six moyennes entreprises canadiennes et 33 experts sont mis à contribution lors des trois itérations menant à la dernière version du prototype.

Compte tenu de leurs caractéristiques, les PME, et en particulier les moyennes entreprises, constituent le type d'organisations visées par le développement de l'outil. Les six PME ont fait l'objet d'autant d'études de cas. Trois PME sont des entreprises de service (analyses médicales [P02S], agence de placement de personnel [P04S], firme de comptables agréés [P06S]) et trois PME sont des entreprises manufacturières (manufacturier d'appareils électriques $[\mathrm{P} 03 \mathrm{M}]$, fabrication de produits métalliques $(\mathrm{P} 05 \mathrm{M})$, fabrication de machinerie spécialisée $[\mathrm{P} 07 \mathrm{M}])$. Le nombre total d'employés des entreprises varient entre 60 et 410 employés. Une autre entreprise [P01S] a également participé au développement initial du questionnaire.

Les experts ont évalué les pratiques des PME à partir d'énoncés de cas et ont commenté la démarche et les résultats des diagnostics. Le nombre d'experts ayant participé à l'une ou l'autre des étapes de la recherche s'élève à 33, dont 15 relativement aux études de cas. Pour chacune des six études de cas, un nombre variant entre sept et neuf experts ont retourné les feuilles de commentaires remplies. 


\section{Résultats et discussion}

Dans un contexte de recherche-action, les résultats de la recherche portent davantage sur la description du travail accompli. Le résultat de la recherche étant le développement et la validation d'un outil informatisé, il s'agit de décrire la démarche. Les résultats décrits dans chaque étape comprennent non seulement les résultats pour la période principale de développement, mais aussi pour l'ensemble du développement. En effet, compte tenu du caractère cyclique de la recherche-action et du prototypage, il est normal que certains éléments développés à un moment précis se voient modifiés ultérieurement par une analyse des résultats.

\section{1. Étapes de la démarche de recherche}

La présente recherche-action, qui permet de développer un prototype de système expert sur les pratiques de la veille stratégique, se divise en cinq étapes:1) l'élaboration de la base de connaissances, 2) le développement et la validation préliminaire du prototype, 3) le développement et la validation du prototype, 4) l'essai du prototype et 5) l'analyse du prototype. Ces étapes se déroulent sur deux périodes distinctes; elles font l'objet d'une description détaillée dans la section subséquente. Il y a lieu de noter que les étapes ne sont pas linéaires mais correspondent davantage à une spirale comme l'illustre le modèle de la recherche-action (Susman et Evered, 1978).

La première étape de l'élaboration de la base de connaissances correspond à la première tentative du chercheur d'acquérir et de représenter la connaissance. L'acquisition de la connaissance s'effectue à partir d'une revue de la documentation et de l'expertise du chercheur et d'autres experts. Il s'agit essentiellement d'établir les problèmes de gestion de la veille stratégique en s'attardant plus particulièrement aux PME. La représentation de la connaissance s'effectue par le développement d'un réseau sémantique (Muhr,1997) et par la production de règles. Pour réaliser le réseau sémantique, le logiciel ATLAS/ti a été utilisé. L'examen systématique des études empiriques a permis de recenser près de 150 études empiriques liées d'une manière quelconque à la veille stratégique. Cet examen a notamment permis de compléter le réseau sémantique et de vérifier l'aspect opérationnel des variables liées à la veille stratégique. Au total, 418 concepts sont répertoriés dans le réseau sémantique portant sur la veille stratégique et ces concepts font l'objet de 539 relations entre eux. L'ensemble des concepts et des variables relevés a servi de base dans l'élaboration du questionnaire et dans l'intégration de ces 
variables dans les règles du système expert. Les relations entre les concepts sont également intégrées dans les règles. Les variables du questionnaire proviennent donc de l'expertise recueillie à cette étape.

La deuxième étape vise le développement et la validation préliminaire du prototype ainsi que la validation de la démarche de recherche. Cette étape correspond au premier essai du chercheur de développer en pratique le prototype de système expert. Il s'agit de concevoir et de rédiger un questionnaire pour recueillir des données afin de rendre explicites les pratiques de veille stratégique sous tous leurs angles et d'alimenter le système expert. Ensuite, il faut s'occuper de la programmation de l'outil informatisé ; le choix technologique et la maîtrise de la programmation du système expert représentent des défis importants à ce moment-là. Enfin, la validation de la démarche de recherche permet de vérifier si le projet tient la route.

Un questionnaire couvrant l'ensemble des thèmes de la veille stratégique répertoriés a été conçu. Comprenant 32 pages, ce questionnaire s'appuie sur l'ensemble des concepts et variables définis lors de la première étape portant sur l'acquisition de connaissances. Le questionnaire se compose de sections permettant de représenter les diverses composantes (voir figure 3) Et le format des réponses correspond presque exclusivement à une échelle de Likert à cinq points $(1,2,3,4,5)$, à une réponse dichotomique (oui/non) ou à un choix dans une liste préétablie. L'annexe I permet d'en visualiser certains extraits.

La troisième étape constitue le cœur du développement et de la validation du prototype. Pour parvenir à développer un prototype fonctionnel, trois itérations utilisant six études de cas ont permis de valider les diverses versions du prototype et d'effectuer ensuite la mise au point du prototype en modifiant les éléments de programmation. Cette étape fait appel simultanément à des entreprises et à des experts. Il s'agit alors d'améliorer le prototype à l'aide d'études de cas et de commentaires provenant des dirigeants de PME et d'experts. L'analyse des résultats après chaque cas permet d'améliorer la mise au point du prototype et de réviser la base des connaissances.

L'ensemble de rapports et documents accompagnant le développement comprennent les données d'un questionnaire spécifique, l'énoncé de cas, le rapport à l'entreprise du diagnostic des pratiques de veille, un document de travail contenant les explications de la logique du système expert et les feuilles de commentaires pour le développement ${ }^{2}$. Les annexes I et II en présentent certains extraits.

2. Il est possible de consulter des exemples des rapports et documents dans Brouard (2004), disponible sur le site Internet de l'auteur <http://sprott.carleton. $\mathrm{ca} / \sim \mathrm{fbrouard} />$. 
Le rapport à l'entreprise du diagnostic des pratiques de veille stratégique constitue le principal produit émis par le système expert; il comporte 22 pages. Ce rapport contient une description de la veille stratégique et de l'outil, un sommaire des signaux (feux de circulation), une brève description de l'entreprise, des sections de diagnostic pour chaque composante du diagnostic (général, types de veille, contexte de la veille, organisation de la veille, processus de la veille, sécurité), le plan d'action / interventions prioritaires, les bénéfices et obstacles perçus de la veille, une annexe expliquant les diagnostics et une table des matières. L'utilisation des formes géométriques jumelées aux couleurs (carré [rouge], losange [jaune] et cercle [vert]) permet de contourner les problèmes d'impression en noir et blanc. Chaque composante du diagnostic comporte des éléments généraux et des constats et recommandations spécifiques, incluant des exemples pratiques à implanter. Produit avec le logiciel Microsoft Word, il est toutefois possible pour l'utilisateur du prototype de modifier ce rapport en ajoutant ou en enlevant certains éléments; il a ainsi le loisir de se servir des différentes parties du rapport. Ce rapport correspond au produit transmis à l'entreprise à la suite de sa participation. L'annexe II permet de visualiser certains extraits du rapport à l'entreprise.

La quatrième étape concerne l'essai du prototype. Durant cette étape, un rapport est émis à chaque entreprise faisant l'objet d'une étude de cas. Avec la production du rapport à l'entreprise, un ou deux dirigeants d'entreprises sont rencontrés afin de recueillir leurs réactions après la présentation du rapport. Cette présentation se déroule lors du deuxième entretien avec les dirigeants de l'entreprise sous étude. Chronologiquement, cette étape s'effectue non pas après la troisième étape mais simultanément. En effet, les cas utilisés pour l'essai visent les mêmes entreprises que pour le développement et la validation du prototype.

La cinquième étape correspond à l'analyse du prototype et elle complète la phase d'évaluation. Durant cette étape, des commentaires sont recueillis auprès des dirigeants d'entreprises ayant participé à l'étude. En plus des réactions émises à la suite de la présentation du rapport lors de la quatrième étape, un entretien supplémentaire permet d'obtenir d'autres commentaires et réactions quelques mois après la présentation du rapport.

\section{2. Études de cas}

La nature cyclique de la recherche-action amène l'utilisation d'itérations afin d'analyser les six études de cas. Si des changements apparaissent nécessaires à la suite de l'analyse des données recueillies pour une étude de cas, des modifications sont réalisées après leur identification. À la fin d'une itération, 
on analyse toutefois l'ensemble des changements requis, afin de s'assurer que ces changements sont effectués avant de passer à l'itération suivante. La plupart du temps, les modifications apportées au prototype consistent en de petits changements ou raffinements à la programmation du système expert. Il ne s'agit donc pas d'éléments majeurs à corriger, mais de nombreux détails qui affectent les résultats produits avec le système expert. Durant les itérations, les experts sont essentiellement ceux qui soulignent les éléments à corriger ou à préciser.

Globalement, la veille stratégique mérite une amélioration dans toutes les PME étudiées. En effet, deux entreprises sont évaluées à un niveau rouge, quatre entreprises, à un niveau jaune et aucune ne se situe au niveau vert. Cet échantillon, correspondant aux évaluations des pratiques de veille dans les études antérieures, indique une évaluation générale des entreprises où les PME manifestent des degrés variés dans leurs pratiques. Une différence peut se retrouver au niveau vert, qui n'est pas représenté dans les entreprises étudiées, alors que des études mentionnent que certaines PME sont très avancées au regard de leurs pratiques de veille stratégique. Cela correspond approximativement à la proportion obtenue par Lesca et Raymond (1993). Les résultats de l'étude du CNRC-ICIST (1999) sur les pratiques de veille stratégique des entreprises canadiennes indiquent pourtant qu'il existe bel et bien des PME qui réalisent des activités de veille de classe mondiale.

Dans l'ensemble, les interventions générales prioritaires sont l'organisation de la veille, le processus de la veille, la sécurité et le contexte de la veille. Les types de veille ne semblent pas poser de problème particulier. Quoique les interventions spécifiques prioritaires varient selon les entreprises, les plus fréquentes parmi les six PME étudiées sont la formalisation de la veille, les mesures de protection et de risques et les ressources de la veille. Il est à noter que quatre entreprises jugent très utile la veille stratégique et une PME la juge assez utile.

\section{Retombées de la recherche}

Cette recherche-action offre plusieurs retombées et apprentissages, autant pour les chercheurs que pour les dirigeants de PME et leurs conseillers. Ces retombées sont conformes aux dimensions mentionnées par Dick (1997) concernant la recherche-action, soit la dimension compréhension (recherche) et la dimension changement (action). La dimension compréhension se concentre sur la démarche de recherche, la base de connaissances et le questionnaire alors que la dimension changement se concentre sur la réalisation de l'outil informatisé, le diagnostic et le rapport remis à l'entreprise. 


\subsection{Retombées pour les chercheurs}

Le développement du prototype a permis de réaliser un outil informatisé sous forme de système expert afin de diagnostiquer les pratiques de veille stratégique de PME. L'outil diagnostique représente l'artefact produit afin d'aider les dirigeants d'une organisation à se sensibiliser aux activités de veille stratégique et à progresser. Plus particulièrement, l'artefact comprend la réalisation d'une base de connaissances, d'un questionnaire et des rapports. Jumelé avec le questionnaire qui alimente le système expert, la base de connaissances constitue l'élément central du système expert. L'ensemble des concepts et variables inclus dans cette base de connaissances présente une synthèse des différentes dimensions des pratiques de veille stratégique.

Le questionnaire qui s'étend sur 32 pages est considéré comme étant complet de l'avis des experts. La contrainte de temps imposant une limite d'environ 60 minutes pour répondre au questionnaire a restreint certaines nuances pouvant être considérées intéressantes par certains intervenants. Le questionnaire peut servir de base pour un chercheur qui voudrait examiner une dimension particulière et non l'ensemble des composantes de la veille stratégique, comme c'est le cas avec le prototype. Par exemple, un chercheur pourrait utiliser uniquement les éléments touchant la collecte et les sources de renseignements.

La démarche de recherche a été très bien reçue tant de la part des dirigeants que de celle des experts. La recherche a permis le développement d'un outil informatisé global et d'envergure des pratiques de veille stratégique des PME.

\subsection{Retombées pour les PME}

L'évaluation des retombées de la présente recherche-action pour les PME s'évalue en termes d'utilité pour les dirigeants de PME. Du point de vue d'un dirigeant de PME, le produit qu'il reçoit de l'utilisation du prototype correspond au rapport à l'entreprise contenant le diagnostic des pratiques de veille stratégique de son entreprise. Ce rapport comprend principalement les signaux pour les 30 composantes, des recommandations pratiques offrant des moyens concrets d'action et une indication des priorités d'action. Ce rapport équilibre les besoins des gens d'affaires en leur permettant de repérer rapidement les éléments importants du rapport, en leur donnant des exemples concrets d'actions et en leur fournissant des explications sur l'évaluation des pratiques.

De l'avis de dirigeants de PME, la sensibilisation à la veille stratégique est «grandement améliorée par la réflexion suscitée par le questionnaire 
et par le rapport à l'entreprise». Selon tous les répondants rencontrés, la réflexion personnelle réalisée en complétant le questionnaire est également bénéfique, non seulement en ce qui a trait à la veille stratégique mais aussi à la gestion en général de leur entreprise. À ce stade de développement, cette sensibilisation est toutefois très limitée au niveau de l'ensemble de l'entreprise. Selon les réactions recueillies, les diagnostics posés avec le prototype semblent être mieux adaptés à certains types d'entreprises.

\subsection{Retombées pour les praticiens de la veille}

Les praticiens de la veille, conseillers externes ou internes à l'entreprise, peuvent également bénéficier de cette recherche; en effet, cet outil constitue un artefact leur permettant de visualiser une méthodologie susceptible d'être empruntée. Les praticiens peuvent aussi comparer leur méthodologie d'évaluation de la veille stratégique avec celle offerte par l'outil diagnostique développé. Ils peuvent ainsi évaluer leur propre méthodologie et la modifier, s'ils le jugent à propos. La base de connaissances constitue en outre une synthèse pratique de la documentation existante traitant de la veille et des conseils d'experts pour l'action des PME. Cette synthèse présente une structure globale des pratiques de veille stratégique où figurent les 30 composantes du diagnostic avec laquelle les intervenants peuvent organiser leurs recommandations et les entreprises, leurs actions et leur rapport.

\section{Conclusion}

En conclusion, la recherche-action permet d'entamer un dialogue avec des dirigeants de PME sur un concept généralement considéré comme abstrait. La nature même du prototype, sa complexité, le temps requis et le faible nombre de cas comparativement à celui nécessaire pour arriver à un système à maturité (Delisle et St-Pierre, 2003) et le nombre limité d'experts peuvent justifier la nécessité de poursuivre le développement. Même si le système expert est applicable à un ensemble de PME, il est indispensable d'apporter des nuances pour certaines industries. Ainsi, la pondération utilisée a été modifiée pour tenir compte d'une différence majeure entre les entreprises de service et manufacturières.

De l'avis des dirigeants de PME ayant participé à la recherche, l'utilité de l'outil diagnostique est indéniable. Répondant favorablement à la question de recherche, l'outil qui en résulte fait ressortir les pratiques existantes de veille stratégique et facilite la sensibilisation au processus de veille stratégique. 
Somme toute, il reste beaucoup à accomplir pour développer les pratiques de veille stratégique dans les organisations, qu'il s'agisse de PME ou d'autres types d'entreprises. L'outil permet de poser des jalons pour continuer l'exploration et faire progresser ces pratiques. La réaction déjà très positive des dirigeants de PME à l'outil est fort encourageante pour l'avenir. En outre, ce constat encouragera peut-être la mise sur pied d'un programme de recherche sur ce thème afin d'explorer ce champ d'expertise important pour aider les organisations à piloter dans leur environnement turbulent.

ANNEXE I

\section{Questionnaire pour réaliser le diagnostic (extrait)}

\section{II.1 Environnement externe}

Parmi les éléments suivants de l'environnement externe, cochez sur une échelle de 1 (faible) à 5 (élevé) le niveau d'importance de chaque élément pour l'entreprise.

\begin{tabular}{|c|c|c|c|c|c|c|c|}
\hline & \multicolumn{2}{|c|}{ faible } & \multicolumn{3}{|c|}{ élevé } & & \\
\hline & 1 & 2 & 3 & 4 & 5 & & \\
\hline - Technologie & 0 & 0 & $\mathrm{O}$ & $\mathrm{O}$ & O & 1318 & 89012345 \\
\hline - Clients & $\mathrm{O}$ & $\mathrm{O}$ & $\mathrm{O}$ & $\mathrm{O}$ & $\mathrm{O}$ & 1321 & 89012345 \\
\hline - Fournisseurs & $\mathrm{O}$ & $\mathrm{O}$ & $\mathrm{O}$ & O & $\mathrm{O}$ & 1322 & 89012345 \\
\hline - Concurrents & $\mathrm{O}$ & 0 & O & O & O & 1326 & 89012345 \\
\hline $\begin{array}{l}\text { - Contexte social } \\
\text { (démographie, écologie, } \\
\text { politique, juridique, } \\
\text { socioculturel, économique) }\end{array}$ & $\mathrm{O}$ & $\mathrm{O}$ & $\mathrm{O}$ & $\mathrm{O}$ & $\mathrm{O}$ & 1331 & 89012345 \\
\hline
\end{tabular}

Évaluez les enjeux suivants en indiquant s'il s'agit ou non d'une menace. Cochez le chiffre approprié sur une échelle de 1 (pas une menace) à 5 (grande menace).

- L'apparition de nouveaux produits

- L'apparition de nouveaux

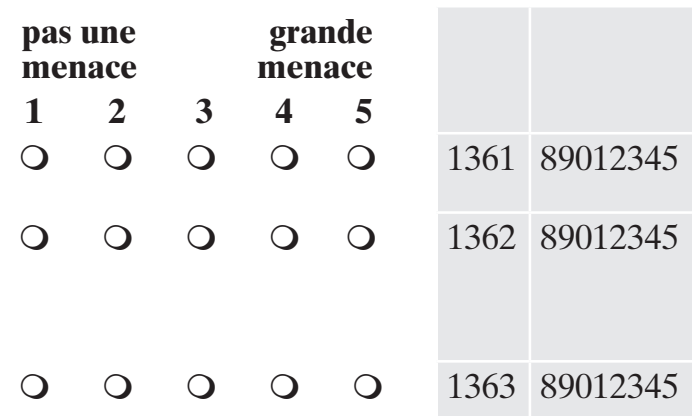
matériaux, processus d'affaires et procédés de fabrication

- Le rétrécissement du marché

\begin{tabular}{|c|c|c|c|c|c|c|c|}
\hline & \multicolumn{2}{|c|}{$\begin{array}{l}\text { pas une } \\
\text { menace }\end{array}$} & \multicolumn{3}{|c|}{$\begin{array}{l}\text { grande } \\
\text { menace }\end{array}$} & & \\
\hline & 1 & 2 & 3 & 4 & 5 & & \\
\hline $\begin{array}{l}\text { - L'apparition de } \\
\text { nouveaux produits }\end{array}$ & 0 & O & 0 & 0 & 0 & 1361 & 89012345 \\
\hline $\begin{array}{l}\text { - L'apparition de nouveaux } \\
\text { matériaux, processus } \\
\text { d'affaires et procédés } \\
\text { de fabrication }\end{array}$ & 0 & 0 & 0 & 0 & O & 1362 & 89012345 \\
\hline $\begin{array}{l}\text { - Le rétrécissement } \\
\text { du marché }\end{array}$ & 0 & 0 & 0 & 0 & 0 & 1363 & 89012345 \\
\hline
\end{tabular}


AnNeXe I

Questionnaire pour réaliser le diagnostic (suite)

\begin{tabular}{|c|c|c|c|c|c|c|c|}
\hline $\begin{array}{l}\text { - La rareté de la } \\
\text { main-d'œuvre }\end{array}$ & $\mathrm{O}$ & $\mathrm{O}$ & $\mathrm{O}$ & O & $\mathrm{O}$ & 1364 & 89012345 \\
\hline $\begin{array}{l}\text { - Les difficultés dans les } \\
\text { approvisionnements }\end{array}$ & $\mathrm{O}$ & $\mathrm{O}$ & $\mathrm{O}$ & O & $\mathrm{O}$ & 1365 & 89012345 \\
\hline $\begin{array}{l}\text { - La concurrence sur les prix, } \\
\text { la qualité ou la nouveauté }\end{array}$ & O & O & $\mathrm{O}$ & $\mathrm{O}$ & $\mathrm{O}$ & 1366 & 89012345 \\
\hline $\begin{array}{l}\text { - La surcapacité de } \\
\text { production dans l'industrie }\end{array}$ & $\mathrm{O}$ & $\mathrm{O}$ & O & O & $\mathrm{O}$ & 1367 & 89012345 \\
\hline $\begin{array}{l}\text { - Un contexte de } \\
\text { ralentissement économique }\end{array}$ & $\mathrm{O}$ & $\mathrm{O}$ & $\mathrm{O}$ & $\mathrm{O}$ & $\mathrm{O}$ & 1368 & 89012345 \\
\hline - Les changements & $\mathrm{O}$ & $\mathrm{O}$ & $\mathrm{O}$ & $\mathrm{O}$ & 0 & 1369 & 89012345 \\
\hline
\end{tabular}

ANNEXE II

\section{Exemple d'un rapport à l'entreprise (extrait)}

\section{SOMMAIRE}

Les signaux lumineux des différentes composantes du diagnostic sont:

\begin{tabular}{lllll}
\hline VEILLE & & & \\
STRATÉGIQUE & & jaune & 50 \\
\hline TYPES DE VEILLE & & $\begin{array}{l}\text { Veille } \\
\text { technologique } \\
\text { Veille } \\
\text { commerciale } \\
\text { Veille } \\
\text { concurrentielle } \\
\text { Veille sociétale }\end{array}$ \\
\hline
\end{tabular}

Revue internationale P.M.E., vol. 20, nº 1, 2007 
AnNeXe II

Exemple d'un rapport à l'entreprise (suite)

\begin{tabular}{|c|c|c|c|}
\hline \multirow[t]{5}{*}{$\begin{array}{l}\text { CONTEXTE } \\
\text { ORGANISATIONNEL }\end{array}$} & & jaune & 50 \\
\hline & Structure de la veille & ert & 90 \\
\hline & Culture de la veille & jaune & 50 \\
\hline & Direction de la veille & vert & 90 \\
\hline & Ressources de la veille & rouge & 10 \\
\hline \multirow[t]{8}{*}{$\begin{array}{l}\text { ORGANISATION } \\
\text { DE LA VEILLE }\end{array}$} & & vert & 90 \\
\hline & Approches de la veille & une & 50 \\
\hline & $\begin{array}{l}\text { Formalisation } \\
\text { de la veille }\end{array}$ & & 90 \\
\hline & Fréquence de la veille & & 90 \\
\hline & Intégration de la veille & ert & 90 \\
\hline & $\begin{array}{l}\text { Diversification } \\
\text { de la veille }\end{array}$ & & 90 \\
\hline & Intensité de la veille & aune & 50 \\
\hline & Éthique de la veille & rouge & 10 \\
\hline \multirow[t]{7}{*}{$\begin{array}{l}\text { PROCESSUS DE } \\
\text { LA VEILLE }\end{array}$} & & jaune & 50 \\
\hline & Cycle & & 90 \\
\hline & Planification & une & 50 \\
\hline & Collecte & aune & 50 \\
\hline & Analyse & aune & 50 \\
\hline & Diffusion & une & 50 \\
\hline & Évaluation & ine & 50 \\
\hline
\end{tabular}

Note $:$ Carré $=$ Niveau rouge $($ Agir $) ;$ Losange $=$ Niveau jaune $($ Améliorer $)$; Cercle $=$ Niveau vert (Poursuivre)

Revue internationale P.M.E., vol. 20, nº 1, 2007 


\section{DIAGNOSTIC TYPES DE VEILLE}

\section{Constats et recommandations}

En général, les types de veille stratégique de l'organisation sont à un niveau vert (90). Un maillon fort de vos activités de veille stratégique se situe dans les types de veille. Il est important de continuer les pratiques existantes.

La veille technologique de l'organisation est à un niveau vert (90).

Il est important de continuer les pratiques existantes au niveau de la veille technologique.

Au besoin, vous pourriez aussi considérer les éléments suivants:

- Préparer un tableau de suivi des technologies.

- S'assurer de la mise à jour régulière du tableau de suivi des technologies.

- Reconnaître l'importance de la technologie qui pourrait amener une rupture dans l'innovation.

La veille commerciale de l'organisation est à un niveau vert (90).

Il est important de continuer les pratiques existantes au niveau de la veille commerciale.

Au besoin, vous pourriez aussi considérer les éléments suivants:

- Préparer un tableau de suivi des clients.

- S'assurer de la mise à jour régulière du tableau de suivi des clients.

- Reconnaître l'importance de suivre les clients qui sont la source des revenus.

- Préparer un tableau de suivi des fournisseurs.

- S'assurer de la mise à jour régulière du tableau de suivi des fournisseurs.

- Reconnaître l'importance de suivre les fournisseurs pouvant affecter la livraison des biens et services.

\section{PLAN D'ACTION / INTERVENTIONS PRIORITAIRES}

Les interventions générales prioritaires sont les suivantes.

Intervention générale $\mathrm{n}^{\circ} \mathrm{I}$ : PROCESSUS DE LA VEILLE (51)

L'entreprise pourrait améliorer son processus de veille stratégique.

Intervention générale $\mathrm{n}^{\circ}$ II : SÉCURITÉ (54)

L'entreprise pourrait améliorer la sécurité de son information.

Intervention générale $\mathrm{n}^{\circ}$ III: ORGANISATION DE LA VEILLE (59)

Revue internationale P.M.E., vol. 20, nº 1, 2007 
L'entreprise pourrait continuer l'implantation de mesures pratiques afin d'organiser efficacement la veille stratégique.

Les interventions spécifiques prioritaires sont les suivantes.

Intervention spécifique $\mathrm{n}^{\mathrm{o}} 1$ : Mesures de protection (5)

Des mesures de protection devraient être mises en place pour assurer la sécurité de l'information dans l'entreprise.

Intervention spécifique $\mathrm{n}^{\circ} 2$ : Éthique de la veille (19)

Les activités de veille stratégique devraient faire plus de place aux considérations éthiques.

Intervention spécifique $\mathrm{n}^{\circ} 3$ : Approches de la veille (26)

Un effort devrait être consenti pour adapter une approche de la veille correspondante aux besoins. 


\section{Bibliographie}

Aguilar, F.J. (1967), Scanning the Business Environment, New York, Macmillan.

Audet, J. (1998), La veille stratégique chez les PME québécoises : une étude de cas par comparaisons intersites, Thèse de doctorat, Québec, Université Laval.

Auster, E. et C.W. Choo (1994), «CEOs, Information and decision making: scanning the environment for strategic advantage », Library Trends, vol. 43, n 2, p. 206-225.

Avenier, M.-J. (2004), «L'élaboration de savoirs actionnables en PME légitimés dans une conception des sciences de gestion comme des sciences de l'artificiel», Revue internationale PME, vol. 17, $\mathrm{n}^{\text {os }} 3$-4, p. 13-42.

Awad, E.M.(1996), Building Expert Systems: Principles, Procedures and Applications, Minneapolis/St. Paul, West Publishing Company.

BASKERVILLE, R. (1999), «Investigating information systems with action research», Communication of the Association for Information Systems, vol. 2, n 19, p. 1-31.

BASKerville, R. et M. Myers (2004), «Special issue on action research in information systems : making IS research relevant to practice - foreword», MIS Quarterly, vol. 28, n 3, p. 329-335.

Baskerville, R.L. et A.T. Wood-Harper (1996), «A critical perspectives on action research as a method for information systems research », Journal of Information Technology, vol. 11, n 3, p. 235-246.

Baskerville, R.L. et A.T. Wood-Harper (1998), «Diversity in information systems action research methods», European Journal of Information Systems, vol. 7, $\mathrm{n}^{\circ}$ 2, p. 90-107.

Benfer, R.A., E.E. Brent et L. Furbee (1991), Expert Systems, Newbury Park, Sage Publications.

Bergeron, P. (1997), «A qualitative case study approach to examine information resources management », Canadian Journal of Information and Library Science,

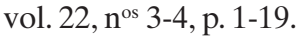

Bergeron, P. (2000), Veille stratégique et PME - Comparaison des politiques gouvernementales de soutien, Québec, Presses de l'Université du Québec.

Beynon-Davies, P., D. Tudehope et H. Mackay (1999), «Information systems prototyping in practice», Journal of Information Technology, vol. 14, p. 107-120.

Brouard, F. (2004), Développement d'un outil diagnostique des pratiques existantes de la veille stratégique auprès des PME, Thèse de doctorat, Trois-Rivières, Université du Québec à Trois-Rivières.

Bulinge, F. (2002), Pour une culture de l'information dans les petites et moyennes organisations: un modèle incrémental d'intelligence économique, Thèse de doctorat, Toulon, Université de Toulon et du Var, Laboratoire LePont.

Bulinge, F. (2003), «L'intelligence économique comme instrument de développement autonome dans les PMO: proposition d'un modèle de transfert», Communication présentée lors de la conférence «Intelligence économique: recherches et applications» (IERA'2003), Nancy, 14-15 avril.

Revue internationale P.M.E., vol. 20, nº 1, 2007 
Chanal, V., H. Lesca et A.C. Martinet (1997), «Vers une ingénierie de la recherche en gestion », Revue française de gestion, $\mathrm{n}^{\circ}$ 116, novembre-décembre, p. 41-51.

CHoo, C.W. (1999), «The art of scanning the environment», Bulletin of the American Society for Information Science, vol. 25, n 3, p. 21-24.

CHоo, C.W. (2001), «Environmental scanning as information seeking and organizational learning» [version électronique], Information Research, vol. 7, $\mathrm{n}^{\circ} 1$, 29 p. Saisie de <http://InformationR.net/ir/7-1/paper112.html>.

Choudhury, V. et J.L. SAmpler (1997), «Information specificity and environmental scanning: an economic perspective», MIS Quarterly, vol.21, n 1, p. 25-53.

CNRC-ICIST (1999), «Une étude des compagnies canadiennes de recherche et de développement - sensibilisation à l'information à valeur concurrentielle et utilisation de cette information dans l'industrie canadienne de la technologie Rapport de deuxième étape: Entrevue et résultats finaux » [version électronique], Saisie de $<$ http://www.nrc.ca/cisti/ref/nrccis_f.html $>$.

Delisle, S. et J. ST-Pierre (2003), «An expert diagnosis system for the benchmarking of SMEs' performance», Proceedings of the First International Conference on Performance Measures, Benchmarking and Best Practices in the New Economy (Business Excellence '03), p.191-196, Guimaraes, Portugal, 10-13 juin.

Dick, B. (1997), Approaching an Action Research Thesis: An Overview, [version électronique], Saisie de $<$ http ://www.scu.edu.au/schools/gcm/ar/arp/phd.html $>$.

DolBec, A. (1998), «La recherche-action», dans B. Gauthier (dir.), Recherche sociale: de la problématique à la collecte des données, p. 467-496, $3^{\mathrm{e}}$ édition, Québec, Presses de l'Université du Québec.

Eden, C. et C. Huxman (1996), «Action research for the study of organizations», dans S.R. Clegg, C. Hardy et W.R Nord (dir.), Handbook of Organization Studies, p. 526-542, Londres, Sage Publications.

EgIDERIa (2003), Saisie le 14 mars 2003 de <http ://www.egideria.fr>.

Eisenhardt, K.M. (1989), «Building theories from case study research», Academy of Management Review, vol. 4, n ${ }^{\circ}$ 4, p. 532-550.

ELENKov, D.S. (1997), «Strategic uncertainty and environmental scanning: the case for institutional influences on scanning behavior », Strategic Management Journal, vol. $18, n^{\circ} 4$, p. 287-302.

Ganesh, U., C.E. Miree et J. Prescott (2003), «Competitive intelligence field research: moving the field forward by setting a research agenda», Journal of Competitive Intelligence and Management, vol. 1, n ${ }^{\circ}$ 1, p. 1-12.

Greenwood, D.J. et M. Levin (1998), Introduction to Action Research, Thousand Oaks, Sage Publications.

GREPME (P.-A. Julien, dir.) (1997), Les PME: bilan et perspectives, GREPME (Groupe de recherche en économie et gestion des PME), $2^{e}$ édition, Québec, Presses Inter Universitaires; Paris, Economica.

HEBERT, F.J.et J.H. BRADLEy (1993), «Expert systems development in small business: a managerial perspective», Journal of Small Business Management, vol. 31, $\mathrm{n}^{\circ} 3$, p. 23-34. 
Hult, M. et S. Lennung (1980), «Towards a definition of action research: a note and bibliography», Journal of Management Studies, vol. 17, mai, p. 241-250.

JACOB, R., P.-A. Julien et L. RAYmond (1997), «Compétitivité, savoirs stratégiques et innovation: les leviers de l'apprentissage collectif en contexte de réseau», Revue internationale de gestion, vol. 22, $\mathrm{n}^{\circ} 3$, p. 93-100.

Julien, P.-A. (dir.) (1994), Pour des PME de classe mondiale, Montréal, Transcontinental.

Julien, P.-A., L. Raymond, R. JacoB et C. Ramangalahy (1999), «Types of technical scanning in manufacturing SMEs: an empirical analysis of patterns and determinants », Entrepreneurship and Regional Development, vol. 11, p. 281-300.

KASARDA, J.D. et D. Rondill (1998), «Innovative infrastructure for agile manufacturers », Sloan Management Review, vol. 39, n 2, p. 73-82.

LARIVET, S. (2002), Les réalités de l'intelligence économique en PME, Thèse de doctorat, Toulon, Université de Toulon et du Var.

LAU, F. (1999), «Toward a framework for action research in information systems studies », Information, Technology \& People, vol. 12, n 2, p. 148-175.

Lavoie, L., D. Marquis et P. Laurin (1996), La recherche-action: Théorie et pratique, Québec, Presses de l'Université du Québec.

LesCA, H. (1991), «Fennec: logiciel expert pour l'évaluation de la veille stratégique dans les PME/PMI», Direction et Gestion, vol. 26, $\mathrm{n}^{\text {os }}$ 132-133, p. 9-15.

LescA, H. (1994), «Veille stratégique pour le management stratégique - État de la question et axes de recherche», Économies et Sociétés, Série Sciences de Gestion SG, vol. 20, n 5 , p. 31-50.

LESCA, H. (2003), Veille stratégique. La méthode L.E.S.CAnning, Colombelles, Éditions EMS.

LESCA, H. et L. RAYMOND (1993), «Expérimentation d'un système-expert pour l'évaluation de la veille stratégique dans les PME», Revue internationale PME, vol. $6, \mathrm{n}^{\circ} 1, \mathrm{p} .49-65$.

Lesca, H. et K. Rouibah (1997), «Des outils au service de la veille stratégique», Systèmes d'information et Management, vol. 2, nº 2, p. 101-131.

LescA, N. (2000), «Processus de construction du sens à partir de signes d'alerte précoce: proposition d'un nouvel outil d'aide à la production de connaissance PUZZLE®5», dans les actes de la IX ${ }^{\mathrm{e}}$ conférence annuelle de l'Association internationale de management stratégique (AIMS), Montpellier, 24-26 mai 2000.

LiU, S. (1998), «Strategic scanning and interpretation revisiting: foundations for a software agent support system - Part 1: understanding the concept and context of strategic scanning», Industrial Management \& Data Systems, vol. 7, p. 295-312.

Lyoussoufyine, K. et L. RAYmOND (1998), «Les systèmes experts: une approche pour assister les dirigeants de petites entreprises », Revue Organisations et territoires, vol. $7, \mathrm{n}^{\circ} 2$, p. 83-92.

Revue internationale P.M.E., vol. 20, nº 1, 2007 
Mansell, G. (1991), «Action research in information systems development»,Journal of Information Systems, vol. 1, p. 29-40.

Martin, W.S., W.T. Jones, E. McWilliams et M.V. Nabors (1991), «Developing artificial intelligence applications : a small business development center case study », Journal of Small Business Management, vol. 29, n 4, p. 28-32.

Moreau, E., L. Raymond, B. BelmaAza et S. Turcot (2002), «Un système d'aide à la décision intelligent pour les PME manufacturières: diagnostic comparatif de la performance en ressources humaines », dans les Actes du VI ${ }^{\mathrm{e}}$ Congrès international francophone sur la PME CIFPME 2002, Montréal, octobre.

Moreau, E., L. Raymond et S. Turcot (2003), «Prototyping an intelligent DSS for a comprehensive and comparative performance diagnosis of manufacturing SMEs », Communication présentée lors du 48th World Conference International Council for Small Business (ICSB), Belfast, 15-18 juin.

Munr, T. (1997), ATLAS/ti Visual Qualitative Data Analysis Management Model Building in Education Research \& Business - User's Manual and Reference, Berlin, Scientific Software Development.

Novasys (1991), Les systèmes experts au Québec: bilan et prospectives, Enquête CRIM91/10-04, Montréal, Centre de recherche informatique de Montréal.

O'BRIEN, J.A. (2001), Introduction aux systèmes d'information: un outil essentiel pour l'entreprise branchée, Montréal, Chenelière/McGraw-Hill.

RAYMOND, L. (2000), «Mondialisation, économie du savoir et compétitivité: un cadre de veille des tendances et des enjeux stratégiques pour la PME», Gestion, vol. $25, \mathrm{n}^{\circ} 2$, p. 29-38.

RAYMOND, L. et H. LESCA (1995), «Evaluation and guidance of environmental scanning in SMEs: an expert systems approach », dans les actes de l'Academy of Business Administration 1995 National Conference, p. 539-546, Reno.

Rubiello, L. (1997), Techniques innovantes en informatique, Paris, Hermes.

Sснмітт, C. (2004), «Pour une approche dialectique de la relation entre recherche et pratiques entrepreneuriales: une relation en quête de sens », Revue internationale PME, vol. 17, n ${ }^{\text {os }} 3-4$, p. 43-68.

Susman, G. et R. Evered (1978), «An assessment of the scientific merits of action research », Administrative Science Quarterly, vol. 23, n 4, p. 582-603.

Turban, E. et J.E. Aronson (1998), Decision Support Systems and Intelligent Systems, $5^{\text {e }}$ édition, Upper Saddle River, Prentice Hall.

VANDENBosCh, B. et S.L. HufF (1997), «Searching and scanning: how executives obtain information from executive information systems », MIS Quarterly, vol. 21, $\mathrm{n}^{\circ} 1$, p. 81-107.

YIN, R.K. (1994), Case Study Research: Design and Methods, 2édition, Thousand Oaks, Sage Publications.

Zou, S. et S.T. CAvUSGIL (1996), «Global strategy: a review and an integrated conceptual framework», European Journal of Marketing, vol. 30, n 1, p. 52-69. 